\title{
Emergence Delirium in children is not related to intraoperative Electroencephalogram suppression - findings from a prospective, observtional study
}

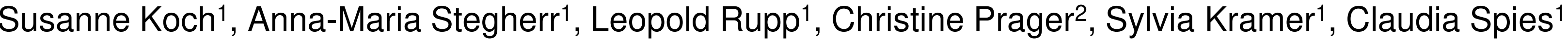 \\ Department of Anaesthesiology and Intensive Care Medicine, Campus Virchow-Klinikum and Campus Charité Mitte, Charité-Universitätsmedizin Berlin, Augustenburger Platz 1, 13353 Berlin, Germany \\ 2Department of Paediatria and Neurology, Campus Virchow-Klinikum and Campus Charité Mitte, Charité-Universitätsmedizin Berlin, Augustenburger Platz 1, 13353 Berlin, Germany
}

\section{Background and Goal of Study}

Emergence delirium (ED) is the most frequent brain dysfunction in children recovering from general anaesthesia. Postoperative delirium is related to prolonged duration of deep anaesthesia including burst suppression periods in elderly1,2. In children depth of anaesthesia as measured by Bispectral Index shows no correlation with ED,3. The aim of this prospective, observational study was to determine whether the occurrence and duration of burst suppression (BS) periods influences the incidence of ED.

\section{Materials and Methods}

Prospective, observational study in children $0.5-8$ years, undergoing elective surgery (EA2/027/15 / NCT02481999). Bodyweight adapted oral premedication with midazolam was administered in all children. Patients received induction/maintanence with i.v. Propofol $(\mathrm{mg} / \mathrm{kg}$ ) and/or mask induction with Sevoflurane (etVol\%) according to clinical needs. Bi-frontal EEG was recorded from start of anaesthesia until extubation, throughout this time period duration of BS was visually analyzed (EEG viewer software / $50 \mu \mathrm{V} / 1 \mathrm{~s} / \mathrm{div}$ ). Depth of anaesthesia as measured by Narcotrend Index was determined. ED was assessed during stay in the recovery room using the Pediatric Assessment of Emergence Delirium (PAED) Score. Values $\geq 10$ were defined as ED.

\section{Results and Discussion}

100 children were included, were 40 children developed ED (ED-group), whereas 60 children did not (Non-ED group). No significant differences in the occurrence (ED 47\% vs. Non-ED 55\%, OR $1.4(0.6 / 3.1) ; p=0.541)$ and duration of BS (ED $475 \pm 538 \mathrm{sec}$ vs. Non-ED $645 \pm 604 \mathrm{sec} ; \mathrm{p}=0.174$; Mann-Whitney-U Test) were

\begin{tabular}{|l|c|c|}
\hline & $\begin{array}{c}\text { Non-ED Group } \\
(\mathbf{n}=60)\end{array}$ & $\begin{array}{c}\text { ED Group } \\
(\mathbf{n}=40)\end{array}$ \\
\hline Age [months] & $50 \pm 31$ & $38 \pm 31$ \\
Gender male / female & $37 / 23$ & $25 / 15$ \\
Procedures & & \\
Clef-lip-palate; Oral / neck surgery & 6 & 13 \\
Inguinal hernia / Circumcision / Orchidopexy / Cystoscopy & 16 & 8 \\
Otorhinolaryngology surgery & 7 & 4 \\
Intraabdominal surgery / long procedures (>60min) & 17 & 6 \\
Limb surgery / short procedures (<60min) & 14 & 9 \\
Midazolam premedication (mg/kg body weight) & $0.63 \pm 0.18$ & $0.67 \pm 0.18$ \\
Induction Agent Sevoflurane / Propofol / mixed & $12 / 27 / 21$ & $9 / 13 / 18$ \\
Propofol Induction [mg/kg body weight] & $4.8 \pm 2$ & $4.6 \pm 1.6$ \\
Sevoflurane Induction [etVol \%] & $4 \pm 1.9$ & $4.4 \pm 2$ \\
Anaesthesia duration (min)* & $99 \pm 38$ & $145 \pm 113$ \\
Burst suppression occurrence (yes / no) & $28 / 32$ & $22 / 18$ \\
Burst suppression duration (sec) & $647 \pm 604$ & $475 \pm 538$ \\
Mean Narcotrend Index value & $46 \pm 13$ & $49 \pm 12$ \\
Table 1: Patients' characteristics. Mann- Whitney-U for continuous data, Chi-square for gender, \\
procedures and Induction agent. * indicates a significant difference <0.05.
\end{tabular}
seen with respect to ED (Fig 1). Depth of anaesthesia did not differ between the groups (mean NI: Non-ED $46 \pm 13$ vs. ED $49 \pm 12$ mean NI; $p=0.597$; Mann-Whitney-U Test). Overall, $50 \%$ of the children displayed BS, occurring mainly within 5min after start of anaesthesia (onset BS: 223sec (123/412)). Mixed anaesthesia induction - Sevoflurane and Propofol - led to an increased incidence of BS (mixed induction 68\%; vs. Sevoflurane 24\% and Propofol 46\%; OR 3.3; 95\% Cl 1.4/7.8), $\mathrm{p}=0.008)$. A higher concentration of oral midazolam premedication was positively correlated with prolonged $B S$ duration $\left(R^{2}\right.$ cubic $=0.133$, $\mathrm{p}=0.021)$.

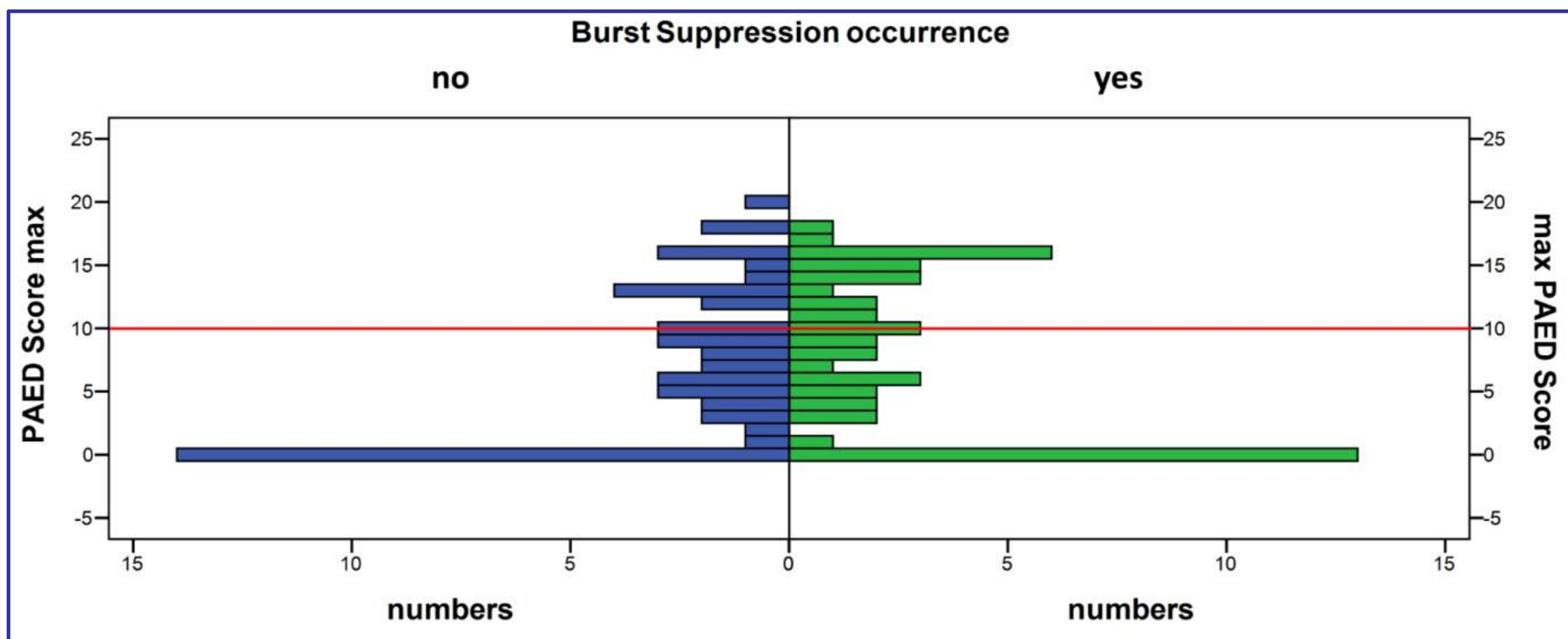

Figure 1: Maximal PAED Score related to occurrence of Burst Suppression. No significant difference in the occurrence of burst suppression was seen with respect to emergence delirium / maximal PAED score values. Emergence delirium was defined as PAED score values $\geq 10$ (see red horizontal line).

\section{Conclusion}

Our data revealed no significant correlation between the occurrence and duration of BS and the incidence of ED. BS, facilitated by a mixed induction with Sevoflurane and Propofol and higher concentrations of premedication with midazolam, does not seem to have an unfavourable impact on cerebral function in children. 